

\section{CRREL Report 85-20}

\section{Constitutive relations for a planar, simple shear flow of rough disks}

Hayley H. Shen and Mark A. Hopkins 


\begin{tabular}{|c|c|}
\hline REPORT DOCUMENTATION PAGE & $\begin{array}{l}\text { READ INSTRUCTIONS } \\
\text { BEFORE COMPLETING FORM }\end{array}$ \\
\hline \begin{tabular}{|l|l|} 
1. REPORT NUMBER & 2. GOVT ACCESSION NO. \\
CRREL RepOrt $85-20$ & \\
\end{tabular} & 3 RECIPIENT'S CATALOG NUMBER \\
\hline \multirow[t]{2}{*}{$\begin{array}{l}\text { 4. TITLE (and Subttie) } \\
\text { CONSTITUTIVE RELATIONS FOR A PLANAR, } \\
\text { SIMPLE SHEAR FLOW OF ROUGH DISKS }\end{array}$} & 5. TYPE OF REPORT \& PERIOD COVERED \\
\hline & 6. PERFORMING ORG. REPORT NLMMER \\
\hline $\begin{array}{l}\text { 7. AUTHOR( } \\
\text { Hayley H. Shen and Mark A. Hopkins }\end{array}$ & 8. CONTRACT OR GRANT NUMBER(B) \\
\hline 9. PERFORMING ORGANIZATION NAME AND ADDRESS & $\begin{array}{l}\text { 10. PROGRAMELEMENT PROJECT, TASK } \\
\text { AREA \& WORK UNIT NUMBERS }\end{array}$ \\
\hline \multirow{2}{*}{$\begin{array}{l}\text { 11. CONTROLLING OFFICE NAME AND ADORESS } \\
\text { U.S. Army Cold Regions Research and } \\
\text { Engineering Laboratory } \\
\text { Hanover, New Hampshire } 03755-1290\end{array}$} & $\begin{array}{l}\text { 12. REPORT DATE } \\
\text { DeCEMber } 1985\end{array}$ \\
\hline & $\begin{array}{l}\text { 13. NUMBER OF PAGES } \\
26\end{array}$ \\
\hline \multirow[t]{2}{*}{ 14. MONITORING AGENCY NAME \& ADDRESS(If different from Controlling Office) } & $\begin{array}{l}\text { 15. SECURITY CLASS. (of thio roport) } \\
\text { Unclassified }\end{array}$ \\
\hline & $\begin{array}{l}\text { 15. DECLASSIFICATION DOWNGRADING } \\
\text { SCHEDULE }\end{array}$ \\
\hline
\end{tabular}

16. DISTRIBUTION STATEMENT (of thio Roport)

Approved for public release; distribution is unlimited.

17. OISTRIBUTION STATEMENT (of the abetract entered in Block 20, it different from Roport)

18. SUPPLEMENTARY NOTES

19. KEY wORDS (Continue on rovorae alde H nocesaary and tdentlly by block number)
Avalanches
Disks
Flow
Granular flow
Slurries

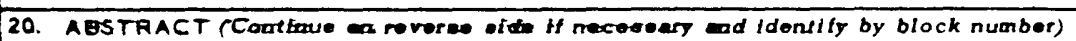

Stresses developed in a rapid, simple shear flow of disks are quantified. Collisional momentum transfer is considered to be the dominant stress generating mechanism. The disks are inelastic and frictional. The restitution coefficient and the coefficient of friction together determine the transfer of momentum and dissipation of energy during a collision. The frictional coefficient generates and maintains a rotational motion of disks. The total fluctuation motion of disks consists of two translational modes a nd one rotational mode. The rotational mode is found to depend on both the restitution and the friction coefficient. Equipartitions of energy among all modes of motion is absent. The mean rotation, however, 


\section{Abstract (cont'd)}

depends only on the mean flow gradient. The analysis assumes a constant magnitude for all fluctuation modes. Comparison with a computer simulated disk flow shows good agreement. This implies that the distribution of velocity magnitude may not be crucial to the quantification of stresses. 


\section{PREFACE}

This report was prepared by Dr. Hayley H. Shen, Assistant Professor, and Mark A. Hopkins, Research Assistant, both of the Department of Civil and Environmental Engineering, Clarkson University, Potsdam, New York. The research effort was supported by the U.S. Army Cold Regions Research and Engineering Laboratory, where the first author spent a year on leave from Clarkson University to work as a Research Physical Scientist in the Snow and Ice Branch.

The authors greatly appreciate the above support. The authors also thank reviewers Dr. N.L. Ackermann and Dr. J.T. Jenkins for their comments and $M$. Hardenberg for his editorial assistance.

The contents of this report are not to be used for advertising or promotional purposes. Citation of brand names does not constitute an official endorsement or approval of the use of such commercial products. 


\section{CONTENTS}

\begin{tabular}{|c|c|}
\hline \multirow{2}{*}{ 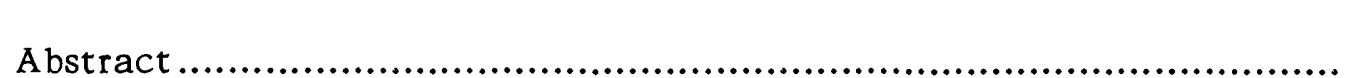 } & \\
\hline & \\
\hline 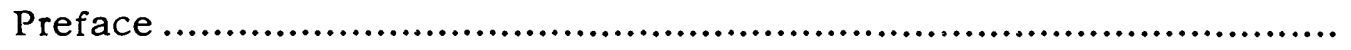 & iii \\
\hline 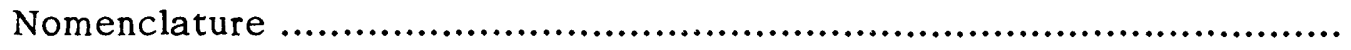 & \\
\hline 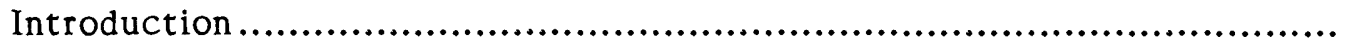 & \\
\hline 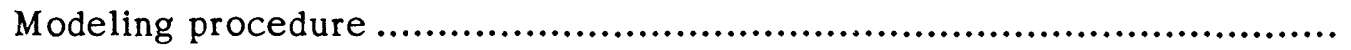 & \\
\hline 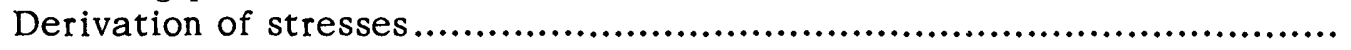 & \\
\hline 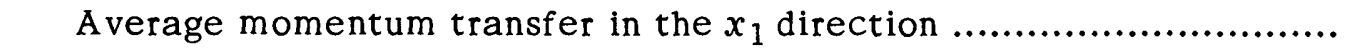 & \\
\hline 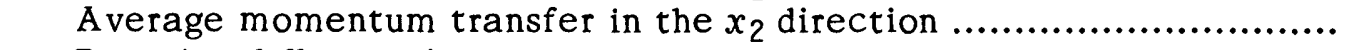 & \\
\hline Rotational fluctuation & \\
\hline 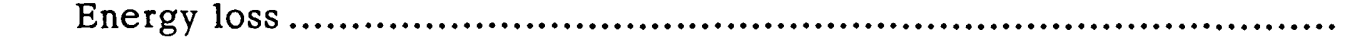 & \\
\hline ..... & 10 \\
\hline esses ...................................... & 11 \\
\hline ...................................... & 11 \\
\hline 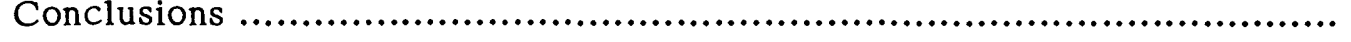 & 12 \\
\hline 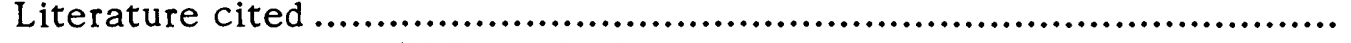 & \\
\hline ocities ................................................. & 15 \\
\hline ppendix B: An alternative method of $\mathrm{fir}$ & \\
\hline
\end{tabular}

\section{ILLUSTRATIONS}

Figure

1. Time lapse image of a gravity-driven disk flow ...................... 2

2. Simple shear flow of uniform disks .......................................... 3

3. Colliding disks moving with mean velocities............................. 4

4. Colliding disks with velocity fluctuations .............................. 5

5. Energy balance in a control area........................................ 5

6. Definition sketch of randomly colliding pairs of disks ................. 6

7. Comparison of theoretical prediction and computer simulation...... 10 


\section{NOMENCLATURE}
$A, B \quad$ Disk identity
$C \quad$ Actual volume concentration of disks
$C_{0} \quad$ Maximum possible disk concentration
D Disk diameter
d Thickness of disks
$E_{\mathrm{R}} \quad$ Rotational energy/ $\frac{1}{2}$ moment of inertia
I Moment of inertia
$\mathbf{i}, \mathbf{j}, \mathbf{k}^{\dagger} \quad$ Unit Cartesian vectors
m Disk's mass
$N \quad$ Areal number density of disks
$\mathbf{N}, \mathbf{P} \quad$ Unit vectors
$p \quad$ Linear number density of disks
$R \quad$ Ratio of the mean velocity to the fluctuating velocity
$s \quad$ Mean interparticle separation distance
$T_{r} \quad$ Fluctuational energy in rotational motion
$T_{\mathrm{t}} \quad$ Fluctuational energy in translational motion
$U_{c} \quad$ Tangential velocity of the contact point
$u \quad$ Mean component of translational velocity
$\nu^{\prime}, v_{A, B}^{\prime} \quad$ Fluctuating component of translational velocity
$\mathbf{V}, \mathbf{V}_{\mathrm{A}, \mathrm{B}}, \mathbf{V}_{\mathrm{B}}$ Total instantaneous translational velocity
$V_{A_{N}} V_{A_{p}}$, Velocity components
$V_{\mathrm{B}_{N}}, V_{\mathrm{B}_{\mathrm{p}}}$
$W \quad$ Work done by shear forces
$x \quad$ Variable
$x_{1}, x_{2} \quad$ Coordinate axes
* Denotes post-collision variables
$\alpha \quad$ Angle between $\mathbf{V}$ and $\mathbf{N}$
$\alpha_{1}, \alpha_{2} \quad$ Integration limits for $\alpha$
B Ratio of the square of the radius of gyration to the square of the radius ( $\frac{1}{2}$ for a disk)
$\overline{\triangle E} \quad$ Average energy loss per collision
$\overline{\Delta M_{1}} \quad$ Average momentum transfer per collision in the $x_{1}$ direction
$\Delta M_{2} \quad$ Average momentum transfer per collision in the $x_{2}$ direction
$\varepsilon \quad$ Coefficient of restitution
$\mu \quad$ Coefficient of friction

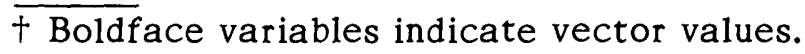


$\rho_{s} \quad$ Density of disks

$\theta \quad$ Angle defining the point of collision with the flow direction

$\Omega \quad$ Mean component of angular velocity

$\omega, \omega_{A}, \omega_{B} \quad$ Instantaneous angular velocity

$\omega^{\prime} \quad$ Fluctuating component of angular velocity

$\Phi \quad$ Dissipation rate from inelastic and frictional collisions

$\tau_{21}, \tau_{22} \quad$ Stresses 


\title{
CONSTITUTIVE RELATIONS FOR A PLANAR, SIMPLE SHEAR FLOW OF ROUGH DISKS
}

\author{
Hayley H. Shen and Mark A. Hopkins
}

\section{INTRODUCTION}

The flow of a granular material is an important transport process in industry as well as in nature. Examples of granular flows are grain flows in chutes and hoppers, slurry flows in pipelines, avalanches, and the transport of sediment and broken ice floes in rivers and oceans. At present, engineers who work with such flows depend largely upon empirical or semi-empirical information. Recent developments in the theories describing the constitutive relationships for rapidly sheared granular flows have revealed many insights into the properties of these flows. These developments may soon lead to a sound theoretical basis for the engineering design of granular flow systems as well as provide a better understanding of many geophysical phenomena.

However, there is a major defect in the existing theories that are capable of analytically describing stresses in rapidly sheared granular flows: these theories neglect the rotational motion of particles. From a laboratory study of gravity flow of rough disks in a frictionless chute with rough walls, * we have observed particle rotation in the shear field and found it to be an important component in the interactions between disks. A sequence of pictures taken from the laboratory study illustrates this phenomenon (Fig. 1).

Recent computer simulations of simple shear flows of rough disks in a channel with rough walls also showed the importance of the particle rotation in the rheological behavior of fast granular flows. Campbell (1982) and Walton (1984) found that the rotation of the disks could contain energy as high as $20 \%$ of the total energy of the disks' motion.

There are a few studies that have considered the rotation of particles (Kanatani 1979, Shahinpoor and Siah 1981). In these studies, the rotation is assumed to be an independent kinematic variable unrelated to its generating mechanisms, which include the material properties and the gradient of the translational velocity field. As a result, only very preliminary, qualitative expressions for the constitutive relationships are obtained, which are of limited engineering interest.

Recently, Lun and Savage (in press) analyzed the effect of particle rotation on the constitutive relations for a granular flow of spheres. They adopted the kinetic theory approach and analyzed the complete dynamics of colliding spheres. They quantified the rotational motion by using the tangential component of the relative velocity and the surface property of the colliding spheres. The surface property of the colliding spheres was assumed to be a material constant $\beta,-1 \leq \beta \leq 1$, such that in the tangential direction of collision, the relative velocity before and after the collision has a ratio equal to $\beta$. The value $\beta=1$ corresponds to a smooth

* Part of an ongoing experimental study at Clarkson University under National Science Foundation Grant No. CME-8011601. 

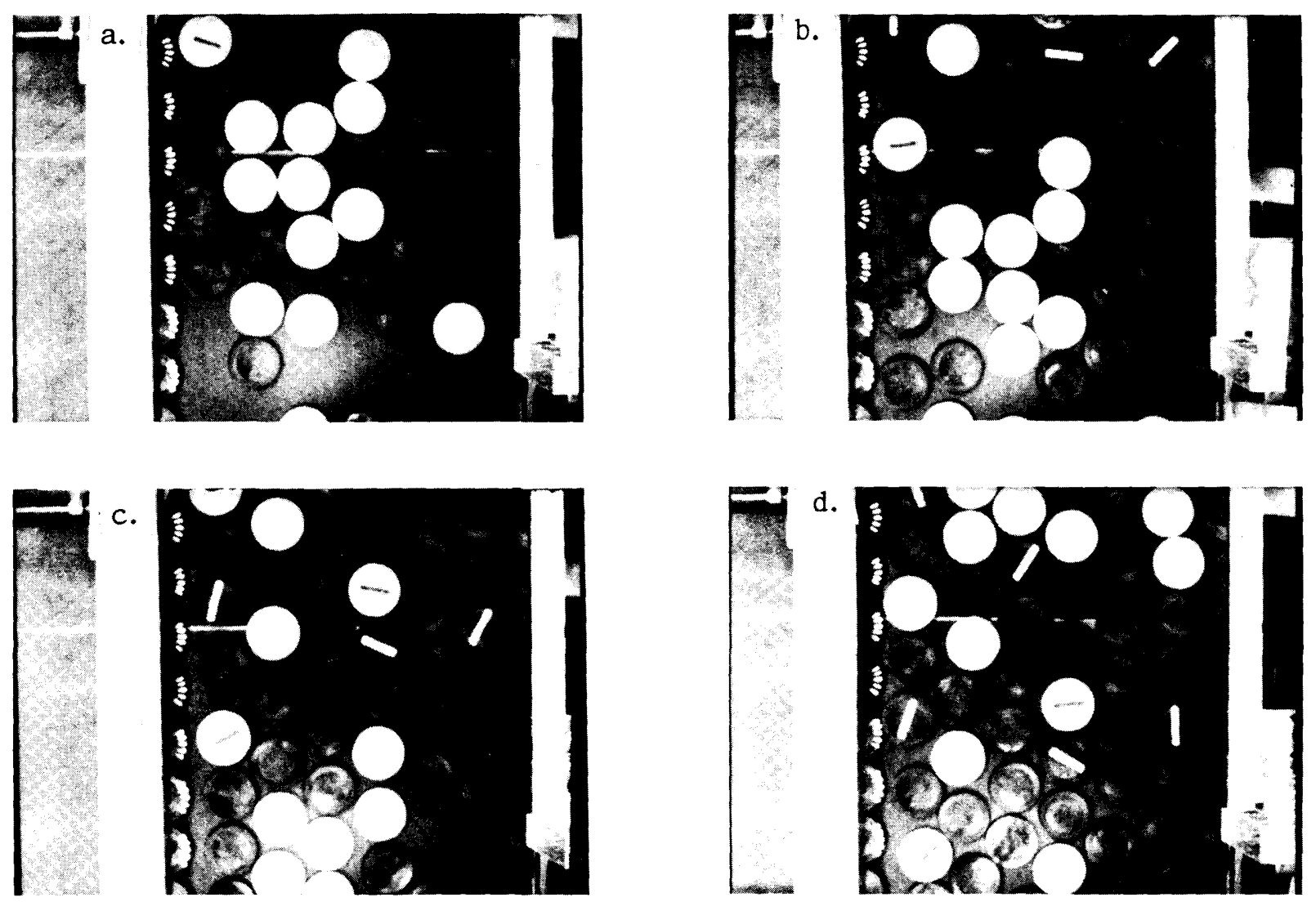

Figure 1. Time lapse image of a gravity-driven disk flow $(\Delta t=0.04 \mathrm{sec})$.

surface with no friction, $\beta=0$ corresponds to infinite friction and $\beta=-1$ corresponds to a complete reversal of tangential velocity. As Lun and Savage (in press) stated, this parameter $\beta$, instead of being a constant, should be a function of the frictional coefficient, the normal impulsive force during collision, and the tangential inelasticity at the contact surface.

In this report, we present an analysis that quantifies the constitutive relations for a planar, simple shear flow of rough disks. This type of flow may be used to model the motion of floating broken ice in northern rivers and arctic seas; therefore the result is of practical interest. Also, there are computer-simulated data (Campbell 1982) for this type of flow that can be used for direct comparison.

The rotational motion of the shearing disks is computed from a statistical average of a random collision process in the granular material. These collisions are inelastic and frictional. It is assumed that all colliding disks separate with no relative tangential velocity. This assumption enables us to directly compare our results with Campbell's data. However, this assumption would mean an infinite friction coefficient. Extension of this analysis to incorporate a finite friction coefficient is currently underway.

The random collision process obtains its energy from the translational mean velocity field. These collisions produce a random motion in the flowing material. This random motion has both translational and rotational modes. Both modes of motion are completely determined by the imposed mean translational velocity field and the material properties. By introducing the restitution and frictional coefficients as independent parameters, the relative motion of colliding particles in the tangential direction can be determined as a function of not only the frictional property, but also the normal impulsive force at contact. This approach incorporates slightly more fundamental physics than that of Lun and Savage (in press). 


\section{MODELING PROCEDURE}

Consider an assembly of shearing uniform disks as shown in Figure 2, where the diameter of disks and the size of the average gap between disks are denoted by $D$ and $s$. The thickness of the disks is $d$, the density of the disks is $\rho_{s}$ and the interstitial fluid is neglected. The area concentration $C$ of the disks is related to $D$ and $s$, as shown in eq 1 , through the densest possible concentration $C_{0}$ (Shen 1982). In this study, $C_{0}$ is assumed to be 0.906 , the concentration for a hexagonal close packing.

$$
\frac{C}{C_{0}}=\left(\frac{D}{D+S}\right)^{2}
$$

The disks in Figure 2 have a restitution coefficient $\varepsilon$ and friction coefficient $\mu$. In our study, $\mu=\infty$ is adopted in order to model collisions such that relative motion at the contact point will be destroyed. This assumption enables us to make a direct comparison with existing computer-simulated data (Campbell 1982) and it greatly simplifies the analysis.

There are four kinematic variables for each individual disk: $u \mathbf{i}$, the mean translational velocity; $\mathbf{v}^{\prime}$, the fluctuation of linear velocity; $\Omega \mathbf{k}$, the mean angular velocity; and $\omega^{\prime}$, the fluctuation of angular velocity. Both the linear and angular fluctuations are random variables that have a spectrum of magnitudes. However, they will be simplified here so that the instantaneous velocities of a disk are

$$
\mathbf{V}=u \mathbf{i}+\mathbf{v}^{\prime}
$$

and

$$
\omega=\Omega \mathbf{k} \pm \omega^{\prime}
$$

where the two fluctuations $\mathbf{v}^{\prime}$ and $\omega^{\prime}$ have constant magnitudes and random directions. The translational velocity $\mathbf{v}^{\prime}$, which occurs in the $x_{1}, x_{2}$ plane, can take all possible directions within the angle $2 \pi$, while the rotations $\omega^{\prime}$ must all be directed along the $\mathbf{k}$ coordinate axis. Therefore

$$
\omega^{\prime}= \pm \omega^{\prime} \mathbf{k} .
$$

The magnitude of the three kinematic variables $\mathbf{v}^{\prime}, \omega^{\prime}$ and $\Omega$ are determined by the material properties of the disks and the driving mechanism created by the shear motion as defined by $u\left(x_{2}\right)$. Without this shearing, collisions can not be maintained in the flow, hence there will be

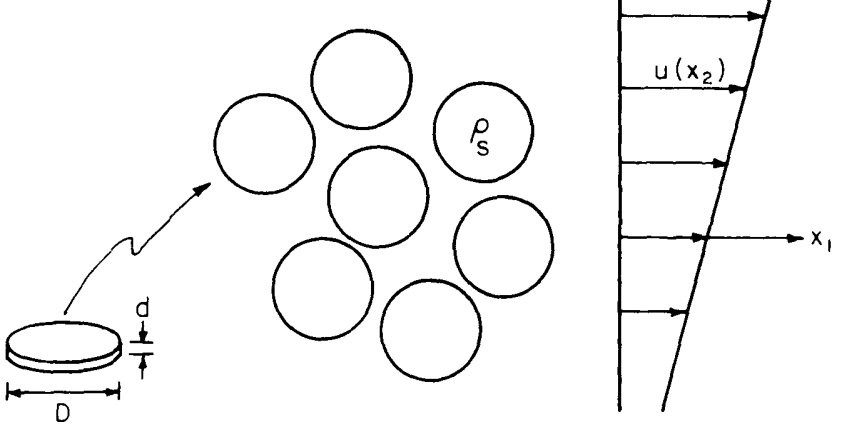

Figure 2. Simple shear flow of uniform disks. no established random motion in either the translational or the rotational sense.

The mean angular velocity $\Omega$ can be obtained through the following analysis. Consider two colliding disks as shown in Figure 3. Since fluctuations have zero net effect on the averaged rotation, these two disks can be assumed to move with the mean velocities as shown in the figure. The relative motion of the two 


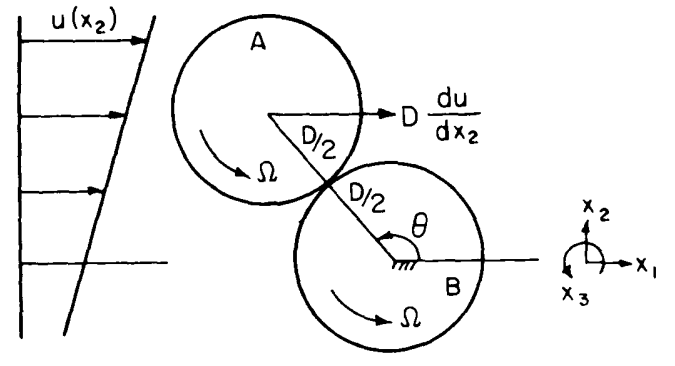

Figure 3. Colliding disks moving with mean velocities.

average sense. Since

$$
\frac{1}{2 \pi} \int_{0}^{2 \pi} \sin ^{2} \theta d \theta=\frac{1}{2}
$$

the average of eq 4 yields

$$
U_{C}=\Omega D+\frac{1}{2} D \frac{d u}{d x_{2}}=0
$$

which implies

$$
\Omega=-\frac{1}{2} \frac{d u}{d x_{2}} \mathbf{k}
$$

This same result has been shown by Campbell (1982) in the computer-simulated shear flow of disks and has been derived by Lun and Savage (in press) for a simple shear flow, assuming that random motions have a Maxwellian distribution. As the derivation of $\Omega$ shows, when $\Omega=-\frac{1}{2}(d u / d x)$, only in the average sense is there no relative motion between two colliding disks. Both the randomness of contact location and relative impact velocity ascribable to disk fluctuation can cause the angular velocity to deviate from the mean value $\Omega$. Denoting the angular velocity fluctuation by $\omega^{\prime}$, this fluctuation is a consequence of random contact location and the fluctuation of the linear velocity $\mathcal{\nu}^{\prime}$.

The angular velocity fluctuation $\omega^{\prime}$ is determined in the following way. The assumption of steady, simple shear flow implies that the energy contained in the rotational mode must be constant. Let

$$
E_{\mathrm{R}}=\omega^{\prime 2} \text {. }
$$

$E_{R}$ being constant requires that, on average, the angular fluctuations before and after collision must be the same. Consider two colliding disks $A$ and $B$ as shown in Figure 4. The angular velocities of these two disks are randomly chosen as

$$
\begin{aligned}
& \omega_{A}=\Omega \pm \omega^{\prime} \\
& \omega_{B}=\Omega \pm \omega^{\prime} .
\end{aligned}
$$

Let $\omega^{*}$ denote the post-collision velocity of disk $B$ from one of the four random combinations of $\omega_{A}$ and $\omega_{B}$. The condition that $E_{R}=\omega^{\prime 2}$ is constant can be 


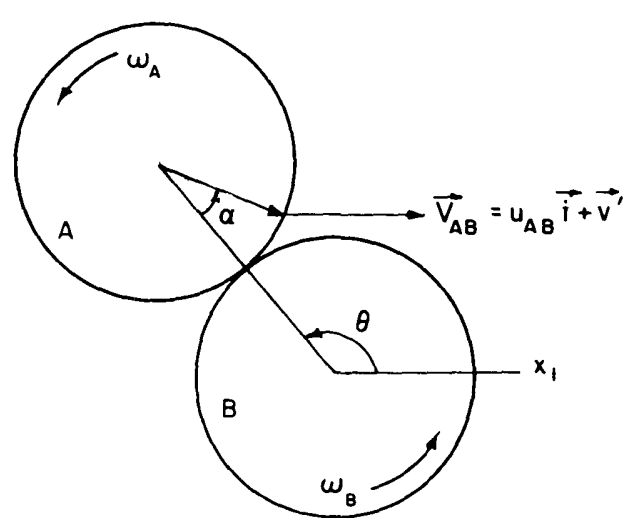

Figure 4. Colliding disks with velocity fluctuations.

described as

$$
\omega^{\prime 2}=\frac{\iint \frac{1}{4} \sum\left(\omega^{*}-\Omega\right)^{2} d \alpha d \theta}{\iint d \alpha d \theta}
$$

where $\theta$ and $\alpha$ are defined in Figure 4 and the integration is over all possible values of these two variables. This integration will be carried out later when ranges for $\theta$ and $\alpha$ are determined. The result of this integration determines $\omega^{\prime}$.

The two kinematic variables, $\nu^{\prime}$ and $\omega^{\prime}$, determine the energetics of this shearing assembly of disks. Consider a control area as shown in Figure 5, where $W$ represents the rate of work done by the shear forces and $\Phi$ represents the rate of dissipation from inelastic and frictional collisions. In a homogeneous flow, as the shearing reaches a steady state, $W$ must be equal to $\Phi$. In mathematical form, this can be written as $W=\Phi$ or

$$
\tau_{21} \frac{d u}{d x_{2}}=N \frac{\nu^{\prime}}{s} \Delta \bar{E}
$$

where $\tau 21$ is the shear force, $N$ is the number of disks in a unit control area, $s$ is the mean gap size between adjacent disks, and $\overline{\Delta E}$ is the average collisional energy loss.

The stresses are modeled as a result of collisional momentum transfer across control surfaces (Bagnold 1954)

$$
\begin{aligned}
& \tau_{21}=p \frac{\nu^{\prime}}{s} \overline{\Delta M}_{1} \\
& \tau_{22}=p \frac{\nu^{\prime}}{s} \overline{\Delta M_{2}}
\end{aligned}
$$

where $p$ is the number of disks per unit length along the boundary of the control area in Figure 5 , and $\overline{\Delta M}_{1}$ and $\overline{\Delta M}_{2}$ are the average linear momentum transfer in the $x_{1}$ and $x_{2}$ directions because of collisions.

As shown by Shen (1982),

$$
p=\frac{4 C}{\pi D}
$$


and

$$
s=D \frac{C_{0}^{\frac{1}{2}}-C^{\frac{1}{2}}}{C^{\frac{1}{2}}}
$$

where $C$ is the area concentration and $C_{0}$ is the densest area concentration that corresponds to $s=0$. Substituting eq 11 into eq 10 and using $p / D=N$ (Shen 1982), we obtain

$$
\frac{d u}{d x_{2}} D \overline{\Delta M}_{1}=\overline{\Delta E} \text {. }
$$

The quantities $\overline{\Delta M}_{1}$ and $\overline{\Delta E}$ are functions of the material properties of the disks and the four kinematic variables $d u / d x_{2}, \Omega, \nu^{\prime}$ and $\omega^{\prime}$. With $\omega^{\prime}$ determined in eq 9 , eq 14 is then used to solve for $\nu^{\prime}$. After $\nu^{\prime}$ and $\overline{\Delta M}_{1}$ are determined, the stresses can then be specified using eq $11 \mathrm{a}$ and $\mathrm{b}$.

In the next section, details are provided regarding the solutions for $\omega^{\prime}, \nu^{\prime}$, $\overline{\triangle M}_{1}$ and $\overline{\Delta M}_{2}$.

\section{DERIVATION OF STRESSES}

In this section, the four averaged quantities-angular velocity fluctuation $\left(\omega^{\prime}\right)$, translational momentum transfer in the $x_{1}$ and $x_{2}$ directions $\left(\overline{\Delta M}_{1}\right.$ and $\left.\overline{\Delta M}_{2}\right)$ and energy loss during collisions $(\overline{\Delta E})$-are first determined as functions of the material properties and the strain rate. Equations $11 \mathrm{a}$ and $\mathrm{b}$ are then used to compute the shear and normal stresses.

As shown in Figure 6, disks $A$ and $B$ are colliding at the location specified by $\theta$. The coordinate system is fixed on disk $B$ and moves with the mean velocity of the disk just prior to collision. The relative fluctuation velocity between $A$ and $B, \sqrt{2 v^{\prime}}$, is randomly distributed between the angles $\alpha_{1}$ and $\alpha_{2}$. The four quantities $\omega^{\prime}, \overline{\Delta M}_{1}, \overline{\Delta M}_{2}$ and $\overline{\Delta E}$ are obtained by integrating over $\theta$ and $\alpha$.

The values of $\alpha_{1}$ and $\alpha_{2}$ are derived from a kinematic constraint on $\nu^{\prime}$, such that the pre-collision velocity of $A, \mathbf{V}_{A B}$, must be within $\pm \pi / 2$ of $\mathbf{N}$ in order for a collision to occur (see Fig. 6). From the law of sines, it can be shown that (Shen

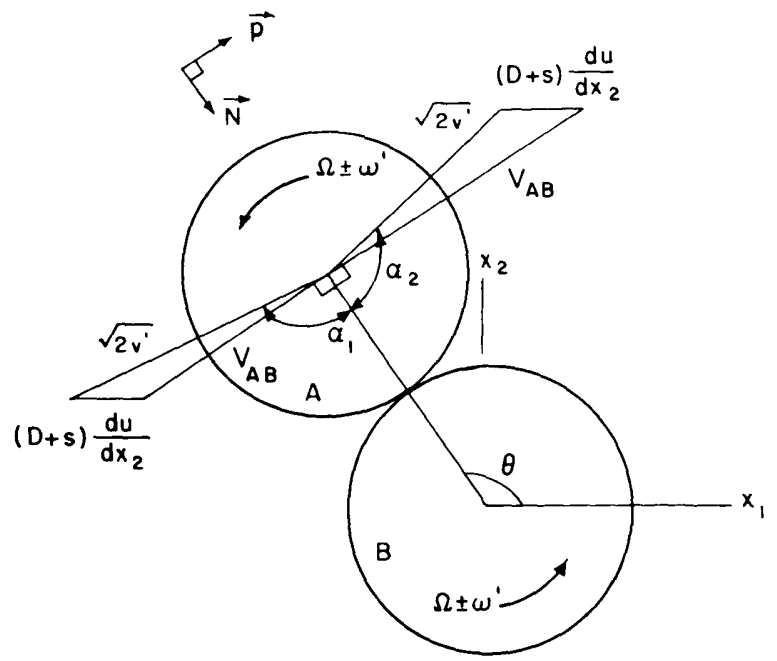

Figure 6. Definition sketch of randomly colliding pairs of disks.
1982)

$$
\alpha_{2}=-\alpha_{1}=\frac{\pi}{2}-\sin ^{-1}(R \sin 2 \theta)
$$

where

$$
R=D \frac{d u}{d x_{2}} / 2 \sqrt{2} \nu^{\prime}
$$

Using the approximation $\sin ^{-1} x \approx x$ yields

$$
\alpha_{2}=-\alpha_{1} \simeq \frac{\pi}{2}-R \sin 2 \theta \text {. }
$$

The unit vectors $\mathbf{N}$ and $\mathbf{P}$ as shown in Figure 6 are the normal and tangential vectors at a given contact point specified by $\theta$. The components of $\mathbf{N}$ and $\mathbf{P}$ are 


$$
\mathbf{N}=-\cos \theta \mathbf{i}-\sin \theta \mathbf{j}
$$

and

$$
\mathbf{P}=\sin \theta \mathbf{i}-\cos \theta \mathbf{j}
$$

The velocity components of disk $A$ prior to the collision are shown in Appen$\operatorname{dix} A$ (eq $A 4 a$ and $A 4 b)$ as

$$
V_{A_{N}}=-D \frac{d u}{d x_{2}} \sin \theta \cos \theta+\sqrt{2} \mathcal{v}^{\prime} \cos \alpha
$$

and

$$
V_{A p}=D \frac{d u}{d x_{2}} \sin ^{2} \theta+\sqrt{2} v^{\prime} \sin \alpha
$$

\section{Average momentum transfer in the $x_{1}$ direction $-\overline{\Delta M}_{1}$}

$\overline{\Delta M}_{1}$ is the average transfer of momentum in the $x_{1}$ direction attributable to collisions. By use of the reference frame that moves with the mean motion of disk $B$ just prior to collision, the transfer of momentum from disk $A$ to disk $B$ becomes equal to the post-collision momentum of disk $B$ in the $x_{1}$ direction. Therefore

$$
\Delta M_{1}=m \mathbf{V}_{\mathrm{B}}^{*} \cdot \mathbf{i}
$$

where $\mathbf{V}_{B}^{*}$ is the post-collision velocity of particle $B$. The average momentum transfer $\overline{\triangle M}_{1}$ resulting from all possible collisions can be expressed as

$$
\overline{\Delta M}_{1}=\rho_{\mathrm{s}} \frac{\pi D^{2} d \int_{0}^{\pi} \int_{\alpha_{1}}^{\alpha_{2}} \Delta M_{1} d \alpha d \theta}{4 \int_{0}^{\pi} \int_{\alpha_{1}}^{\alpha} d \alpha d \theta}
$$

where $\alpha_{1}$ and $\alpha_{2}$ are defined in eq 15. The velocity $V_{B}^{*}$ can be described in terms of the components in the $\mathbf{N}$ and $\mathbf{P}$ directions:

$$
\mathbf{V}_{\mathrm{B}}^{*}=V_{\mathrm{B}_{\mathrm{N}}}^{*} \mathbf{N}+V_{\mathrm{Bp}}^{*} \mathbf{P}
$$

The post-collision velocities of disks $A$ and $B$ depend on the pre-collision velocities and the material properties characterized by the restitution coefficient $\varepsilon$ and the friction coefficient $\mu$. In this analysis, the post-collision tangential velocity between $A$ and $B$ is assumed to be zero. This is the same condition used in Campbell's computer-simulated shear flow of disks (1982) and implies that the friction coefficient $\mu$ is infinite.

Letting $\mu$ equal infinity, the velocity components of disk $B$ after the collision are derived in Appendix A (eq A 5 c and A $5 \mathrm{~d}$ ) as

$$
\begin{aligned}
& V_{\mathrm{B}_{\mathrm{N}}}^{*}=\frac{(1+\varepsilon)}{2}\left(-D \frac{d u}{d x_{2}} \sin \theta \cos \theta+\sqrt{2} \nu^{\prime} \cos \alpha\right) \\
& V_{\mathrm{B}_{\mathrm{P}}}^{*}=\frac{1}{6}\left\{D \frac{d u}{d x_{2}} \sin ^{2} \theta+\sqrt{2} \nu^{\prime} \sin \alpha+\frac{D}{2}\left(\omega_{\mathrm{A}}+\omega_{\mathrm{B}}\right)\right\} .
\end{aligned}
$$


The angular velocities $\omega_{A}$ and $\omega_{B}$ contain both mean and fluctuating components, as defined in eq $2 \mathrm{~b}$. However, since the average of the fluctuating components is zero, $\overline{\Delta M}$ does not contain the fluctuation component of angular velocities. Hence only the average angular velocities are necessary to describe $V_{\mathrm{Bp}_{\mathrm{p}}^{*}}^{*}$ From eq 6 it is seen that

$$
\omega_{A}=\omega_{B}=\Omega=-\frac{1}{2} \frac{d u}{d x_{2}}
$$

therefore, $V_{B p}^{*}$ can be written as

$$
V_{B p}^{*}=\frac{1}{6}\left(-\frac{1}{2} D \frac{d u}{d x_{2}} \cos 2 \theta+\sqrt{2} \nu^{\prime} \sin \alpha\right) \text {. }
$$

After substituting eq $18 \mathrm{a}$ and $\mathrm{b}, 21$ and $A 5 \mathrm{c}$ and d into eq 20 and integrating, we obtain the average momentum transfer in the $x_{1}$ direction as

$$
\overline{\Delta M}_{1}=\rho_{\mathrm{s}} \frac{\pi D^{2}}{4} d\left(D \frac{d u}{d x_{2}}\right)(0.106 \varepsilon+0.124) \text {. }
$$

\section{Average momentum transfer in the $x_{2}$ direction $-\overline{\Delta M}_{2}$}

$\overline{\Delta M}_{2}$ is the average transfer of momentum in the $x_{2}$ direction ascribable to disk collisions. Again our reference frame is fixed on disk B prior to a collision. In this reference frame, the momentum transferred is equal to the post-collision momentum of disk B in the $x_{2}$ direction. Hence the average value of the momentum transfer in the $x_{2}$ direction is

$$
\overline{\Delta M}_{2}=\rho_{\mathrm{s}} \frac{\pi D^{2} d \int_{0}^{\pi} \int_{\alpha_{1}}^{\alpha_{2}} \mathbf{V}_{\mathrm{B}}^{*} \cdot \mathbf{j} d \alpha d \theta}{4 \int_{0}^{\pi} \int_{\alpha_{1}}^{\alpha_{2}} d \alpha d \theta}
$$

with $\alpha_{1}$ and $\alpha_{2}$ defined in eq 15 .

Äfter substituting eq $18 \mathrm{a}$ and $\mathrm{b}, 21$ and $A 5 \mathrm{c}$ and $\mathrm{d}$ into eq 24 and integrating, we find that the average momentum transfer in the $x_{2}$ direction becomes

$$
\overline{\Delta M}_{2}=-\rho_{s} \frac{\pi D^{2} d}{4} \frac{1}{\nu^{\prime}}\left\{0.012\left(D \frac{d u}{d x_{2}}\right)^{2}+0.287(1+\varepsilon) \nu^{\prime 2}\right\} .
$$

\section{Rotational fluctuation-w'}

The instantaneous angular velocity $\omega$ has both a mean and a fluctuating component as defined earlier in eq $2 \mathrm{~b}$. Since all rotations are in the $\mathbf{k}$ direction, $\omega$ may be written as

$$
\omega=\Omega \pm \omega^{\prime} .
$$

Consider two colliding disks $A$ and $B$ as shown in Figure 6 . Let $\omega^{*}$ denote the post-collision angular velocity. As shown in Appendix A (eq A $2 e$ and $f$ with $\beta=\frac{1}{2}$ ),

$$
\omega_{A}^{*}=\omega_{B}^{*}=\frac{1}{3}\left(2 \omega_{A}-\omega_{B}-2 V_{A_{P}} / D\right)
$$

where $\omega_{A}$ and $\omega_{B}$ are given in eq 26 . Since there are four arbitrary combinations of $\omega_{A}$ and $\omega_{B}$, the post-collision angular fluctuations are 


$$
\omega_{B}^{*}-\Omega=\frac{1}{3}\left\{ \pm 2 \omega^{\prime} \pm \omega^{\prime}-2\left(\Omega+\frac{V_{A p}}{D}\right)\right\}
$$

where the four combinations of the \pm signs are to represent the four combinations of $\omega_{A}$ and $\omega_{B}$.

From Appendix A (eq A4b)

$$
V_{A p}=D \frac{d u}{d x_{2}} \sin \theta+\sqrt{2} \nu^{\prime} \sin \alpha
$$

The average angular fluctuations as derived in eq 9 can now be obtained after substituting eq 28 and $A 4 \mathrm{~b}$ into eq 9. The integration limits are again $0 \leqq \theta \leqq \pi$ and $\alpha_{1} \leqq \alpha \leqq \alpha_{2}$, with $\alpha_{1}, \alpha_{2}$ given in eq 17. This integration then gives

$$
\omega^{\prime 2}=0.125\left(\frac{d u}{d x_{2}}\right)^{2}+1.024\left(\frac{\nu^{\prime}}{D}\right)^{2}
$$

This derivation for $\omega^{\prime}$ is rather formal. A more intuitive way of deriving $\omega^{\prime}$ is given in Appendix B, where a semi-Monto-Carlo method is used to generate $\omega^{\prime}$. The result, however, is identical to eq 29.

\section{Energy loss $-\overline{\Delta E}$}

$\overline{\Delta E}$ is the average energy loss in an inelastic and frictional collision. As discussed earlier (eq 7), the average rotational energy does not change in a steady state. Hence $\triangle E$ may be modeled as the result of translational energy loss alone. For a given collision, the translational energy loss in a pair of disks A and B is

$$
\Delta E_{T}=\frac{1}{2} m\left(\mathbf{V}_{A} \cdot \mathbf{V}_{A}-V_{A}^{*} \cdot \mathbf{V}_{A}^{*}+\mathbf{V}_{B} \cdot \mathbf{V}_{B}-\mathbf{V}_{B}^{*} \cdot \mathbf{V}_{B}^{*}\right)
$$

where $m=\rho_{s}\left(\pi D^{2} d / 4\right)$ is the disk's mass.

The average translational energy loss $\overline{\Delta E}_{\mathrm{T}}$ is

$$
\overline{\Delta E_{\mathrm{T}}}=\frac{1}{2} m \frac{\int_{0}^{\pi} \int_{\alpha_{1}}^{\alpha_{2}}\left(V_{A_{N}}^{2}+V_{A_{P}}^{2}-V_{A_{N}}^{* 2}-V_{A_{P}}^{* 2}-V_{B_{N}}^{* 2}-V_{B_{P}}^{* 2}\right) d \alpha d \theta}{\int_{0}^{\pi} \int_{\alpha}^{\alpha_{2}} d \alpha d \theta}
$$

since $\mathbf{V}_{B}$ is zero in the chosen reference frame. As derived in Appendix $A$ (eq $A 5 a$ and $b$ ),

$$
\begin{aligned}
& V_{A_{N}}^{*}=\frac{(1+\varepsilon)}{2}\left(-D \frac{d u}{d x_{2}} \sin \theta \cos \theta+\sqrt{2} \nu^{\prime} \cos \alpha\right) \\
& V_{A_{P}}^{*}=\frac{\left(D \frac{d u}{d x_{2}}\right)\left(5 \sin ^{2} \theta+\frac{1}{2}\right)+5 \sqrt{2} \nu^{\prime} \sin \alpha}{6}
\end{aligned}
$$

Substituting eq $A 4 a$ and $b$ and $A 5 a, b, c$ and d into eq 31 yields the average collisional energy loss

$$
\overline{\Delta E}=\left(D \frac{d u}{d x_{2}}\right)^{2}\left(0.0464-0.0261 \varepsilon^{2}\right)+\mathcal{\nu}^{\prime 2}\left(0.386-0.244 \varepsilon^{2}\right) .
$$


In a steady, homogeneous shear flow, the work done by shear forces must equal the energy dissipation from inelastic and frictional effects of the collisions between disks. This balance law has been shown to reduce to (eq 14)

$$
D \frac{d u}{d x_{2}} \overline{\Delta M}_{1}=\overline{\Delta E}
$$
velocity

Substituting eq 23 and 32 into eq 14, we obtain the fluctuation of linear

$$
v^{\prime}=\left(D \frac{d u}{d x_{2}}\right)\left(\frac{0.0261 \varepsilon^{2}+0.106 \varepsilon+0.0776}{0.386-0.244 \varepsilon^{2}}\right)^{1 / 2}
$$

Substitution of eq 33 into eq 29 allows the flucutation of angular velocity to become

$$
\omega^{\prime}=\frac{d u}{d x_{2}}\left(\frac{-0.0038 \varepsilon^{2}+0.109 \varepsilon+0.128}{0.372-0.244 \varepsilon^{2}}\right)^{1 / 2}
$$

At this point all the kinematic variables have been found in terms of the mean velocity gradient.

\section{SHEAR AND NORMAL STRESSES}

The shear and normal stresses are obtained by substituting eq 23,25 , and 33 into eq $11 \mathrm{a}$ and $\mathrm{b}$. This yields

$$
\tau_{21}=\rho_{s} C\left(\frac{D}{s}\right)(D+s)^{2}\left(\frac{d u}{d x^{2}}\right)^{2}(0.106 \varepsilon+0.124)\left(\frac{0.0261 \varepsilon^{2}+0.106 \varepsilon+0.0076}{0.330-0.244 \varepsilon^{2}}\right)^{1 / 2}
$$

and

$$
\tau_{22}=\rho_{s} C\left(\frac{D}{s}\right)(D+s)^{2}\left(\frac{d u}{d x_{2}}\right)^{2} \frac{0.0075 \varepsilon^{3}+0.035 \varepsilon^{2}+0.053 \varepsilon+0.027}{0.386-0.244 \varepsilon^{2}}
$$

Removing the dimensions from the stresses by dividing eq $35 \mathrm{a}$ and $\mathrm{b}$ by $\rho_{s} C_{0} D^{2}\left(d u / d x_{2}\right)^{2}$ results in the following nondimensional stresses,

$$
\tau_{21}^{*}=\frac{(0.106 \varepsilon+0.124)}{\left[\left(C_{0} / C\right)^{1 / 2}-1\right]}\left(\frac{0.0261 \varepsilon^{2}+0.106 \varepsilon+0.0776}{0.386-0.244 \varepsilon^{2}}\right)^{1 / 2}
$$

and

$$
\tau_{22}^{*}=\frac{-1}{\left[\left(C_{0} / C\right)^{1 / 2}-1\right]} \frac{0.0075 \varepsilon^{3}+0.035 \varepsilon^{2}+0.053 \varepsilon+0.027}{0.386-0.244 \varepsilon^{2}} .
$$

\section{COMPARISON WITH COMPUTER-SIMULATED STRESSES}

To date, there have been no data obtained from physical models of shearing disks that can be used to verify the theory developed above. The only experimental data currently available are from computer simulations by Campbell (1982). The comparison between Campbell's data, eq 40a and b and the shear 

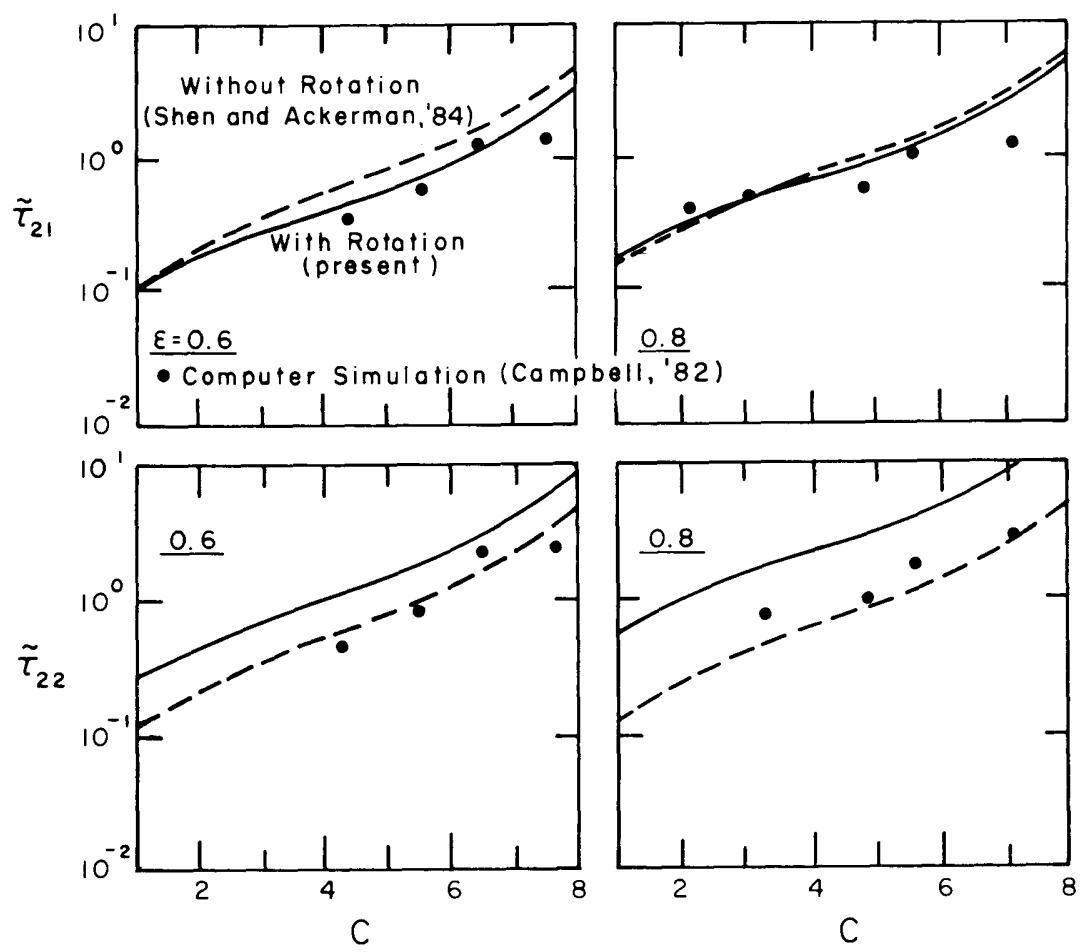

Figure 7. Comparison of theoretical prediction and computer simulation.

stress obtained by Shen (1982), which did not consider disk rotation, is presented in Figure 7.

We observe that by including disk rotations, the shear stress is reduced for the case when $\mu=\infty$. The comparison between the computer simulation data and eq $36 \mathrm{a}, \mathrm{b}$ is, in general, satisfactory except when the disk concentration is lower than 0.3. We believe that at lower concentrations, the momentum transfer that is related to mass transfer increases. However, in the present analysis, mass transfer is not considered. Since for high concentrations, mass transfer is negligible, the correlation is closer in this regime. As the concentration decreases, we therefore expect that collisional momentum transfer alone would produce stresses that are lower than measured values.

\section{DISCUSSION}

We make two major observations on the result of this theoretical development. The first is that the assumption of equipartition of fluctuation energy does not apply in a dissipative granular flow. The second is that the distribution function of the random fluctuating states does not have a significant effect on stresses.

Since the modeling procedure for determining the stresses is very similar to the molecular dynamic approach used with dense gasses, and since the equipartition of energy in molecular random motion is well-known for gasses that are in equilibrium, it has been suggested that a form of equipartition between the rotation and translation motion of each individual disk may exist (Kanatani 1979). It is therefore interesting to compute the ratio between fluctuation energy in the rotational motion and that in the translational motion, which yields 


$$
\frac{T_{r}}{T_{\mathrm{t}}}=\frac{\frac{1}{2} I \omega^{\prime 2}}{\frac{1}{2} m v^{\prime 2}}
$$

where $I$ is the moment of inertia for disks. Substituting eq 29 and 33-and using $I / m=D^{2} / 8$ for a disk-into eq 37 gives us

$$
\frac{T_{r}}{T_{t}}=\left(\frac{0.06-0.04 \varepsilon^{2}}{0.3 \varepsilon^{2}+1.1 \varepsilon+0.8}\right)+0.128
$$

which is far from being equal to unity and depends explicitly on the material property $\varepsilon$. The reason that the friction property does not appear in eq 38 is that the condition of $\mu$ approaching infinity is assumed in this analysis. We expect that when a finite friction coefficient is considered, the partition ratio would also be a function of the friction coefficient.

In this theoretical development, it is recognized that both the linear and angular velocities of disks are random variables. However, in order to make the mathematical manipulation manageable, serious simplifications have been made. We assumed that the fluctuation components $\nu^{\prime}$ and $\omega^{\prime}$ are randomly directed, but constant in magnitude. In order to make a constant $\nu^{\prime}$ and $\omega^{\prime}$ contain the same energy as a distributed $\nu^{\prime}$ and $\omega^{\prime}$, the constant $\nu^{\prime}$ and $\omega^{\prime}$ must be the standard deviations of the real distributions. It has been suggested that the calculated stress level could be lowered significantly by ignoring the real distributions (which could be Maxwellian as suggested by Jenkins and Savage [1983] and Lun et al. [1984]) and by assuming a constant magnitude for the fluctuating components. The stresses obtained by Shen and Ackerman (1982), for example, when analyzing the simple shear flow of uniform spheres were about three-fold lower than those obtained by Lun et al. (1984).

However, the result of the present analysis suggests that this is not the case, at least for a flow of disks.

This conclusion is based upon the close agreement between the present theoretical results and the computer-simulated data of Campbell (1982), since in the computer simulation, the distribution of fluctuation motion is inherently included. If the velocity distribution can make a three-fold magnification of stresses, it would also be revealed in Campbell's data. The fact that the present theory and Campbell's data agree so well supports the validity of this simple model.

\section{CONCLUSIONS}

Constitutive relations are developed for a granular continuum undergoing a simple shear flow. The granular continuum flow consists of uniform disks moving in a plane. The result of the analysis may be used to model the motion of broken ice fields on rivers and oceans.

The major contribution of this analysis is that it includes the disk rotations. The analysis was able to relate this rotation entirely to its generating mechanisms, namely, the linear velocity gradient. Previous studies of this problem either ignored the rotation completely (e.g. Shen and Ackermann 1982, Jenkins and Savage 1983), or treated rotation as an independent mode of motion, unrelated to the linear velocity gradient (e.g., Kanatani 1979, Shahinpoor and Siah 1981).

The stresses are formulated as the rate of momentum transfer attributable to binary collisions. These collisions are inelastic and frictional. The results show explicitly the effect of material properties on the stress level. However, the constitutive laws remain in the second power form 


$$
\tau \sim\left(\frac{d u}{d x_{2}}\right)^{2}
$$

which makes granular flow behave differently from viscous fluid flows.

The significant findings of this investigation are:

1. The stress level is decreased from previous analysis (Shen and Ackermann 1982) when disk rotating is included.

2. There is no equipartition of rotation energy and translational energy.

3. The distribution of random fluctuation does not have significant effect on stresses.

Good agreement between the theoretical results and computer-simulated data is obtained. This demonstrates the need for continued development of the simple model used in this analysis. Future work should extend the present model to analyze mixtures of different sizes and irregular shapes. In order to analyze a general flow, the analysis should also be extended to include a general deformation field described by a tensor with all four components $\partial u_{1} / \partial x_{1}, \partial u_{1} / \partial x_{2}$, $\partial u_{2} / \partial u_{1}, \partial u_{2} / \partial u_{2}$. The above-mentioned studies are currently underway.

\section{LITERATURE CITED}

Bagnold, R.A. (1954) Experiments on gravity-free dispersion of large solid spheres in a Newtonian fluid under shear. Proceedings of the Royal Society of London, Series A, 225: 49-63.

Campbell, C.S. (1982) Shear flow of granular materials. Ph.D. Dissertation. Pasadena: California Institute of Technology.

Jenkins, J.T. and S.B. Savage (1983) A theory for the rapid flow of identical, smooth, nearly elastic, spherical particles. Journal of Fluid Mechanics, 130: 187-202.

Kanatani, K.I. (1979) A micropolar continuum theory for the flow of granular materials. International Journal of Engineering Science, 17: 419-432.

Lun, C.K.K., S.B. Savage, D.J. Jefferey and N. Chepurniy (1984) Kinetic theories for granular flow: Inelastic particles in Couette flow and slightly inelastic particles in a general flow field. Journal of Fluid Mechanics, 140: 223-256.

Lun, C.K.K. and S.B. Savage (In press) A simple kinetic theory for granular flow of rough, inelastic, spherical particles. Journal of Applied Mechanics.

Shahinpoor, M. and J.S.S. Siah (1981) New constitutive equations for the rapid flow of granular materials. Journal of Non-Newtonian Fluid Mechanics, 9: 147156.

Shen, H.H. (1982) Constitutive relationships for fluid-solid mixtures. Ph.D. Dissertation. Potsdam, New York: Clarkson University.

Shen, H.H. and N.L. Ackermann (1982) Constitutive relationships for fluidsolid mixtures. ASCE, Journal of the Engineering Mechanics Division, 108(EM5): 748-763.

Walton, O.R. (1984) Granular flow project. Quarterly report, October-December 1984, Lawrence Livermore National Laboratory, 20 pp. 


\section{APPBNDIX A: POST-COLLISION VELOCITIES}

Consider two disks A and B colliding with each other as shown in Figure 6. Equations governing the collision between disks $A$ and $B$ were developed using the laws of conservation of linear and angular momentum, the energy loss because of incomplete restitution, as specified by the coefficient of restitution, and a frictional closure condition.

$$
\begin{aligned}
& V_{A_{N}^{*}}^{*}+V_{B_{N}^{*}}^{*}=V_{A_{N}}+V_{B_{N}} \\
& V_{A_{p}^{*}}^{*}+V_{B_{p}}^{*}=V_{A_{p}}+V_{B_{p}} \\
& \beta \omega_{A}-\frac{V_{A p}}{R}=\beta \omega_{A}^{*}-\frac{V_{A p}^{*}}{R} \\
& \beta \omega_{B}+\frac{V_{A_{p}}}{R}=\beta \omega_{B}^{*}+\frac{V_{B p}^{*}}{R} \\
& \left(V_{B_{N}}^{*}-V_{A_{N}^{*}}^{*}\right) /\left(V_{B_{N}}-V_{A_{N}}\right)=-\varepsilon \\
& V_{A p}^{*}+R \omega_{A}^{*}=V_{B p}^{*}-R \omega_{B}^{*} .
\end{aligned}
$$

The above six equations are used to solve for the six post-collision variables, $V_{A_{N}^{*}}^{*} V_{A p}^{*}, V_{B_{N}^{*}}^{*}, V_{B_{p}}^{*}, \omega_{A}^{*}$, and $\omega_{B}^{*}$. The frictional closure condition used in this report is that the relative tangential velocity at the point of contact after the collision is zero. This implies that friction is always great enough to destroy the relative tangential velocity, or that friction coefficient is infinite. The six post-collision variables are obtained from eq $A 1 a-f$ as

$$
\begin{aligned}
& V_{A_{N}^{*}}^{*}=\frac{(1-\varepsilon)}{2} V_{A_{N}}+\frac{(1+\varepsilon)}{2} V_{B_{N}} \\
& V_{A_{P}^{*}}^{*}=\frac{(\beta+2) V_{A_{P}}+\beta V_{B_{P}}-D \beta\left(\omega_{A}+\omega_{B}\right) / 2}{2(1+\beta)} \\
& V_{B_{N}^{*}}^{*}=\frac{(1+\varepsilon)}{2} V_{A_{N}}+\frac{(1-\varepsilon)}{2} V_{B_{N}} \\
& V_{B P}^{*}=\frac{\beta V_{A_{P}}+(\beta+2) V_{B_{P}}+D \beta\left(\omega_{A}+\omega_{B}\right) / 2}{2(1+\beta)} \\
& \omega_{A}^{*}=\frac{(1+2 \beta) \omega_{A}-\omega_{B}-2\left(V_{A_{P}}-V_{B_{P}}\right) / D}{2(1+\beta)} \\
& \omega_{B}^{*}=\frac{-\omega_{A^{+}}(1+2 \beta) \omega_{B}-2\left(V_{A_{P}}-V_{B_{P}}\right) / D}{2(1+\beta)} .
\end{aligned}
$$

$\beta$ is the ratio of the square of the radius of gyration to the square of the disk radius. For a uniform, circular disk, $\beta=\frac{1}{2}$.

With the reference frame located at the center of disk $B$ prior to a collision as shown in Figure 6, the pre-collision velocity of disk $B$ is $\mathbf{V}_{B}=0$. Therefore, eq $A 2 a-f$ can be simplified to 


$$
\begin{aligned}
& V_{A_{N}^{*}}^{*}=\frac{(1-\varepsilon)}{2} V_{A_{N}} \\
& V_{A_{P}}^{*}=\frac{1}{6}\left(5 V_{A_{P}}-\frac{D}{2}\left(\omega_{A}+\omega_{B}\right)\right) \\
& V_{B_{N}^{*}}^{*}=\frac{(1+\varepsilon)}{2} V_{A_{N}} \\
& V_{B_{P}}^{*}=\frac{1}{6}\left(V_{A_{P}}+\frac{D}{2}\left(\omega_{A}+\omega_{B}\right)\right) \\
& \omega_{A}^{*}=\frac{1}{3}\left(2 \omega_{A}-\omega_{B}-\frac{2 V_{A_{P}}}{D}\right) \\
& \omega_{B}^{*}=\frac{1}{3}\left(-\omega_{A}+2 \omega_{B}-\frac{2 V_{A_{P}}}{D}\right) .
\end{aligned}
$$

From Shen (1982)

$$
\begin{aligned}
& V_{A_{N}}=-D \frac{d u}{d x_{2}} \sin \theta \cos \theta+\sqrt{2} \nu^{\prime} \cos \alpha \\
& V_{A_{P}}=D \frac{d u}{d x_{2}} \sin ^{2} \theta+\sqrt{2} \nu^{\prime} \sin \alpha .
\end{aligned}
$$

Substituting these into eq A3a-f, we obtain

$$
\begin{aligned}
& V_{A_{N}}^{*}=\frac{(1-\varepsilon)}{2}\left(-D \frac{d u}{d x_{2}} \sin \theta \cos \theta+\sqrt{2} \nu^{\prime} \cos \alpha\right) \\
& V_{A_{P}}^{*}=\frac{1}{6}\left(5 D \frac{d u}{d x_{2}} \sin ^{2} \theta+5 \sqrt{2} \nu^{\prime} \sin \alpha-\frac{D}{2}\left(\omega_{A^{+}}+\omega_{B}\right)\right) \\
& V_{B_{N}}^{*}=\frac{(1+\varepsilon)}{2}\left(-D \frac{d u}{d x_{2}} \sin \theta \cos \theta+\sqrt{2} \nu^{\prime} \cos \alpha\right) \\
& V_{B_{p}}^{*}=\frac{1}{6}\left(D \frac{d u}{d x_{2}} \sin ^{2} \theta+\sqrt{2} \nu^{\prime} \sin \alpha+\frac{D}{2}\left(\omega_{A}+\omega_{B}\right)\right) \\
& \omega_{A}^{*}=\omega_{B}^{*}=-\frac{1}{3} \frac{d u}{d x_{2}}\left(\frac{1}{2}+2 \sin ^{2} \theta\right)+2 \sqrt{2}\left(\nu^{\prime} / D\right) \sin \alpha .
\end{aligned}
$$

In the process of averaging the following series expansions were used:

$$
\begin{aligned}
& \sin x=x-\frac{x^{3}}{3 !}+\frac{x^{5}}{5 !}-\frac{x^{7}}{7 !}+\ldots \\
& \cos x=1-\frac{x^{2}}{2 !}+\frac{x^{4}}{4 !}-\frac{x^{6}}{6 !}+\ldots
\end{aligned}
$$

where $x=\alpha_{2}=\pi / 2-R \sin 2 \theta$ and $R=D\left(d u / d x_{2}\right) / 2 \sqrt{2} \nu^{\prime}$. The series were truncated after the $R^{2}$ term. 


\section{APPENDIX B: AN ALTERNATIVE METHOD OF FINDING $\omega^{\prime}$}

We have defined the post-collision angular velocity of a disk to be

$$
\omega^{*}=\Omega \pm \omega^{\prime} .
$$

Using eq $\mathrm{A} 3 \mathrm{e}$, we can define a "second generation" post-collision angular velocity to be

$$
\omega_{A}^{* *}=\frac{1}{3}\left(2 \omega_{A}^{*}-\omega_{B}^{*}-\frac{2 V_{A p}}{D}\right)
$$

Substituting eq $\mathrm{B} 1$ into eq $\mathrm{B} 2$, and subtracting the mean component, $\Omega$, yields a "second generation" fluctuating angular velocity, which we then square

$$
\omega_{A}^{\prime 2}=\left\{\frac{1}{3}\left[-2\left(\Omega+\frac{V_{A_{P}}}{D}\right) \pm 2 \omega_{A}^{\prime} \pm \omega_{B}^{\prime}\right]\right\}^{2} \text {. }
$$

If we begin with $\omega_{A}^{\prime}=\omega_{B}^{\prime}=0$ (first generation), we find $\omega_{A}^{\prime}$ to be a function of $\left(\Omega+V_{A p} / D\right)$, hence each succeeding generation of $\omega^{\prime}$ is also a function of $\left(\Omega+V_{A p} / D\right)$, which can thus be moved outside the brackets. This quantity is a function of the point of collision denoted by $\theta$ and the direction of $\nu^{\prime}$ denoted by $\alpha$ and is averaged by integration over the range $\theta$ and $\alpha$.

We iterate eq B3 over all previous values of $\omega^{\prime}$ using the following expression

$$
\overline{\omega_{n}^{\prime 2}}=\frac{1}{n^{2}} \sum_{i=0}^{n-1} \sum_{j=0}^{n-1}\left[\frac{1}{3}\left(-2 \pm 2 \omega_{A_{j}} \pm \omega_{B_{j}}^{\prime}\right)\right]^{2} \frac{\iint\left(\Omega+\frac{V_{A_{p}}}{D}\right)^{2} d \alpha d \theta}{\iint d \alpha d \theta}
$$

where $\omega_{A_{j}}^{\prime}=\omega_{B_{j}}^{\prime}=0$ for $i=j=0$.

As $n$ grows large ( 50) the bracketed expression approaches unity. Evaluating the integral expression yields

$$
\omega^{\prime 2}=0.125\left(\frac{d u}{d x_{2}}\right)^{2}+1.024\left(\frac{\nu^{\prime}}{D}\right)^{2}
$$

which is identical to the result obtained by the method outlined previously in eq 29. 